\title{
The role of endoscopic tumor length in resected esophageal squamous cell carcinoma: a retrospective study
}

\author{
Peng Chen ${ }^{1 \#}$, Yuzhen Zheng ${ }^{2 \#}$, Hao He ${ }^{1}$, Pei Yuan Wang ${ }^{1}$, Feng Wang ${ }^{1}$, Shuo Yan Liu ${ }^{1}$ \\ ${ }^{1}$ Department of Thoracic Surgery, Fujian Medical University Cancer Hospital, Fujian Cancer Hospital, Fuzhou, China; ${ }^{2}$ Department of Thoracic \\ Surgery, The Sixth Affiliated Hospital, Sun Yat-sen University, Guangzhou, China \\ Contributions: (I) Conception and design: P Chen, Y Zheng; (II) Administrative support: SY Liu, F Wang; (III) Provision of study materials \\ or patients: All authors; (IV) Collection and assembly of data: P Chen, Y Zheng; (V) Data analysis and interpretation: P Chen, Y Zheng; (VI) \\ Manuscript writing: All authors; (VII) Final approval of manuscript: All authors. \\ "These authors contributed equally to this work. \\ Correspondence to: Shuo Yan Liu. Department of Thoracic Surgery, Fujian Medical University Cancer Hospital, Fujian Cancer Hospital, Fuzhou \\ 350014, China. Email: shuoyanliu2010@163.com.
}

Background: In esophageal squamous cell carcinoma (ESCC), tumor status is assessed on the basis of latitudinal invasion. Endoscopic tumor length (ETL) may represent the longitudinal scope of the primary tumor, and whether it affects tumor stage or prognosis is not entirely clear. In this study, we evaluated the role of ETL in patients with resected ESCC.

Methods: The relationships of ETL with pathological parameters (pT status and $\mathrm{pN}$ status) and overall survival (OS) were analyzed using data from patients with resected ESCC who were treated at Fujian Cancer Hospital between January 1997 and December 2013. Odds ratios (ORs) and hazard ratios (HRs) were fitted with locally weighted scatterplot smoothing, and the structural breakpoints for ETL were determined using the Chow test.

Results: A total of 721 patients with resected ESCC were enrolled. As the ETL increased in these patients, a rise in the risk of advanced pT status, nodal metastasis, and mortality was observed. Cutpoint analysis showed a breakpoint of $7.0 \mathrm{~cm}$. A negative impact of ETL $\geq 7.0 \mathrm{~cm}$ was also found (adjusted HR, 1.335; 95\% CI, 1.004-1.774). Seven independent prognostic factors, including sex, age, number of nodes dissected, $\mathrm{T}$ stage, $\mathrm{N}$ stage, tumor location, and ETL, were identified and entered into the nomogram. The calibration curves for 1-, 3-, and 5-year OS showed optimal agreement between nomogram prediction and actual observation (c-index: 0.688).

Conclusions: Longer tumor length, with ETL $\geq 7.0 \mathrm{~cm}$ as the breakpoint, is a negative prognostic factor in patients with ESCC.

Keywords: Endoscopic tumor length (ETL); esophageal cancer; prognostic factor

Submitted Oct 09, 2020. Accepted for publication Jan 13, 2021.

doi: $10.21037 /$ jtd-21-108

View this article at: http://dx.doi.org/10.21037/jtd-21-108

\section{Introduction}

Despite increased screening and treatment progress, esophageal cancer remains the leading cause of death worldwide. In 2018, there were 572,034 new cases of esophageal cancer diagnosed and 508,585 related fatalities (1). The emergence of multimodality treatments, including immunotherapy and targeted therapy, has seen considerable improvements in the long-term survival of patients with various solid tumors, such as melanoma and non-small cell lung cancer (2-4). However, for patients with localized esophageal squamous cell carcinoma (ESCC), 
the survival rate remains unsatisfactory (5-7). Therefore, novel prognostic factors need to be identified so that patients at high risk of late staging or poor prognosis can be pinpointed.

ESCC is staged according to the tumor, node, and distant metastasis (TNM) system (8). T status, which is defined by tumor infiltration, represents the latitudinal invasion of a primary tumor. At the same time, the endoscopic tumor length (ETL) reflects a primary tumor's longitudinal scope. Theoretically, Tumor depth of invasion and longitudinal scope can more accurately assess the severity of the primary tumor. Biologically, it is plausible that longitudinal growth in the lymphatic-rich submucosa of the esophagus may be a more important factor contributing to regional lymph node metastasis than tumor depth in a single area (9). Nevertheless, the role of ETL has yet to be well clarified (10). Moreover, the methods for identifying cut points are not sufficiently robust (10-14).

In the present study, we set out to evaluate the impact of the longitudinal scope of ESCC on prognosis through an analysis of data from a single institution and to further confirm the relationships of ETL with long-term survival, primary tumor status, and node metastasis.

We present the following article in accordance with the STROBE reporting checklist (available at http://dx.doi. org/10.21037/jtd-21-108).

\section{Methods}

\section{Patients}

Patients who received treatment for ESCC in the Department of Thoracic Surgery at Fujian Cancer Hospital between January 1997 and December 2013 were enrolled into this study. Ethical approval was obtained from the review board committee of Fujian Cancer Hospital. The study was conducted in accordance with the Declaration of Helsinki (as revised in 2013). Because of the retrospective nature of the research, the requirement for informed consent was waived. The inclusion criteria for patients included: (I) pathologically diagnosed ESCC; (II) underwent McKeown-type esophagectomy; (III) pathologic T status of T1, T2, T3, or T4a; (IV) without visceral metastasis (M1 status); (V) microscopically complete resection (R0). The following patients were excluded: patients for whom preoperative endoscopy was necessary; patients with tumor stenosis that was impassable with an endoscope; patients with multiple primary tumor lesions; and patients who had received neoadjuvant anti-cancer therapy. Pathologic staging was reassessed based on the $8^{\text {th }}$ American Joint Committee on Cancer (AJCC) staging system (15).

ETL was determined on the basis of endoscopic results, using the algorithm (distal distance of ESCC - proximal distance of ESCC). Standard endoscopic tumor length (SETL) is defined as the exact ETL value rounded to the nearest integer. To guarantee the reliability of the data, all preoperative endoscopy procedures in this study were performed exclusively in the Department of Endoscopy of Fujian Cancer Hospital.

\section{Adjuvant treatment}

Adjuvant treatment options were selected based on the patient's tumor stage, physical status, and desire, as well as the opinion of the treating physician. In most cases, adjuvant treatment began 4-8 weeks after the operation. Chemotherapy was typically administered as 4-6 cycles of a platinum-based, 2-drug regimen. Patients with R0 resection do not routinely undergo postoperative radiotherapy.

\section{Statistical analysis}

To determine the breakpoint for ETL, the exact ETL value was firstly rounded to the nearest whole numberknown as the "standard endoscopic tumor length (SETL)". The curves of odds ratios [(ORs) $\mathrm{pT}$ status and $\mathrm{pN}$ status] and hazard ratios [(HRs) overall survival (OS)] of each SETL compared with a reference SETL were fitted by using locally weighted scatterplot smoothing (LOWESS) with a bandwidth of $2 / 3$ (default) in $\mathrm{R}$ version 3.2.2 (Bell Laboratories, Murray Hill, NJ; https://cran.r-project.org/ bin/windows/base/old/3.2.2) (16). Structural breakpoints were then determined with SAS 9.3 software using the Chow test (17). The breakpoints were considered to be the threshold of clinical impact.

Statistical analyses were performed using the SPSS 22.0 software package (IBM, inc., Chicago, IL). A 2-sided P value of $<0.05$ was considered to represent statistical significance. The correlations of ETL with patient clinicopathological characteristics were assessed using Pearson's $\chi^{2}$ test. OS was defined as the time from the date of surgery to the date of death or final follow-up. Patients who were lost to followup or still alive at the end of follow-up were censored. The survival rate was calculated using the Kaplan-Meier method, and differences between curves were assessed using 
Table 1 Patient characteristics

\begin{tabular}{|c|c|}
\hline Characteristics & Training cohort, $\mathrm{n}(\%)$ \\
\hline Total & 721 \\
\hline \multicolumn{2}{|l|}{ Sex } \\
\hline Male & $556(77.1)$ \\
\hline Female & $165(22.9)$ \\
\hline Age (year) & $58.9 \pm 8.7$ \\
\hline No. of resected nodes & $28.7 \pm 17.0$ \\
\hline Endoscopic tumor length (cm) & $4.7 \pm 2.1$ \\
\hline \multicolumn{2}{|l|}{ Tumor location } \\
\hline Upper esophagus & $93(12.9)$ \\
\hline Middle esophagus & $411(57.0)$ \\
\hline Lower esophagus & $217(30.1)$ \\
\hline \multicolumn{2}{|l|}{ pT status } \\
\hline $\mathrm{T} 1$ & $82(11.4)$ \\
\hline $\mathrm{T} 2$ & $152(21.1)$ \\
\hline T3 & $457(63.4)$ \\
\hline $\mathrm{T} 4 \mathrm{a}$ & $30(4.2)$ \\
\hline \multicolumn{2}{|l|}{ pN status } \\
\hline NO & 355 (49.2) \\
\hline N1 & $221(30.7)$ \\
\hline N2 & $113(15.7)$ \\
\hline N3 & $32(4.4)$ \\
\hline \multicolumn{2}{|l|}{ Cell differentiation } \\
\hline Well & $142(19.7)$ \\
\hline Moderately & $378(52.4)$ \\
\hline Poorly & $201(27.9)$ \\
\hline \multicolumn{2}{|l|}{$8^{\text {th }}$ AJCC staging system } \\
\hline IA & $7(1.0)$ \\
\hline IB & $80(11.1)$ \\
\hline IIA & $117(16.2)$ \\
\hline IIB & $198(27.5)$ \\
\hline IIIA & $183(25.4)$ \\
\hline IIIB & $88(12.2)$ \\
\hline IIIC & $48(6.7)$ \\
\hline \multicolumn{2}{|l|}{ Adjuvant chemotherapy } \\
\hline No & $613(85.0)$ \\
\hline Yes & $108(15.0)$ \\
\hline
\end{tabular}

AJCC, American Joint Committee on Cancer; pT status, pathological tumor status; $\mathrm{pN}$ status, pathological nodal status. the log-rank test. Factors with potential significance $(\mathrm{P}<0.1)$ in univariate Cox regression analysis were incorporated into multivariate analysis. Multivariate analysis was performed using the Cox proportional hazards model with the backward logistic regression stepwise procedure for the selection of variables.

Based on the results of the multivariable analysis, a nomogram was formulated using the survival and rms packages in R 3.3.2 (http://www.r-project.org) (18). The nomogram was subjected to 1,000 bootstrap resamples for internal validation of the primary cohort. Calibration of the nomogram for 1-, 3-, and 5-year OS was performed by comparing the predicted survival with the observed survival following correction for bias (19). C-indexes were calculated using R 3.2.2 software, with a larger c-index value indicating better predicted precision (20).

\section{Results}

\section{Patient characteristics}

A total of 721 patients who met the eligibility criteria were recruited, including 556 males and 165 females. The enrollees included 7 patients with stage IA, 80 patients with stage IB, 117 patients with stage IIA, 198 patients with stage IIB, 183 patients with stage IIIA, 88 patients with stage IIIB, and 48 patients with stage IIIC disease. The baseline characteristics of the patients are listed in Table 1. The median ETL was $5.0 \mathrm{~cm}$ (range, 1.0-13.0 cm).

\section{Cutpoint analysis for ETL}

Figure 1 shows the fitting curves and corresponding structural breakpoints of SETL for the ORs of T stage and $\mathrm{N}$ stage, and the HR of OS.

All the breakpoints were essentially in agreement (ranging from 5 to 8 ). As survival is the most important outcome, we selected the structural breakpoint of overall survival as the cut point. For generalizability and representativeness, we used $7.0 \mathrm{~cm}$ as the cutoff (Figure 2). As shown in Table 2, a significant association was found between ETL and $\mathrm{pN}$ status $(\mathrm{P}=0.030)$, but in the validation set, this association was not significant.

Among the 721 patients, there were 126 (17.5\%) with an ETL $\geq 7.0 \mathrm{~cm}$. Through the univariate and multivariate analyses, several independent prognostic factors were identified including age, number of nodes dissected, $\mathrm{pT}$ status, pN status, tumor location, and ETL (Table 3). 
A
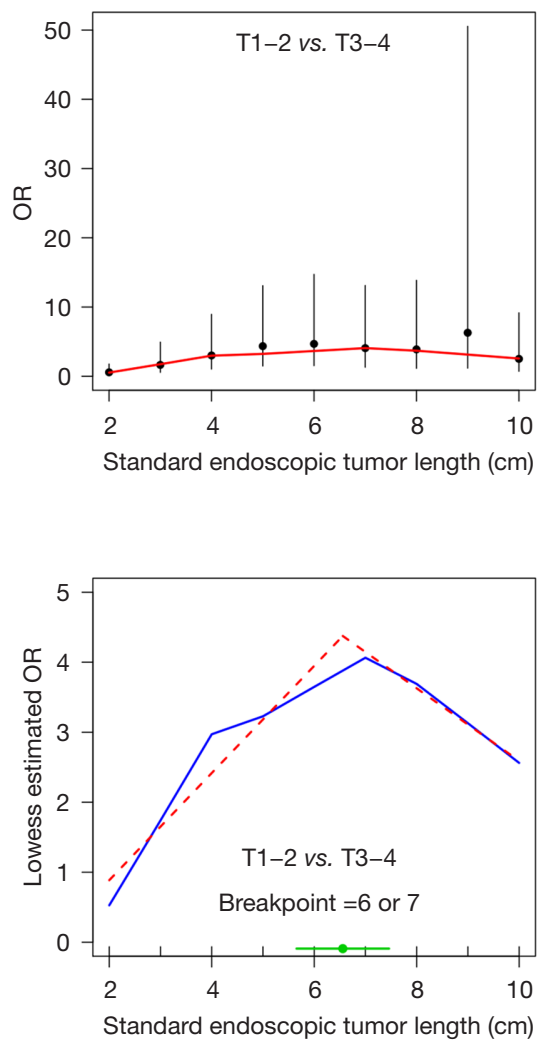

B
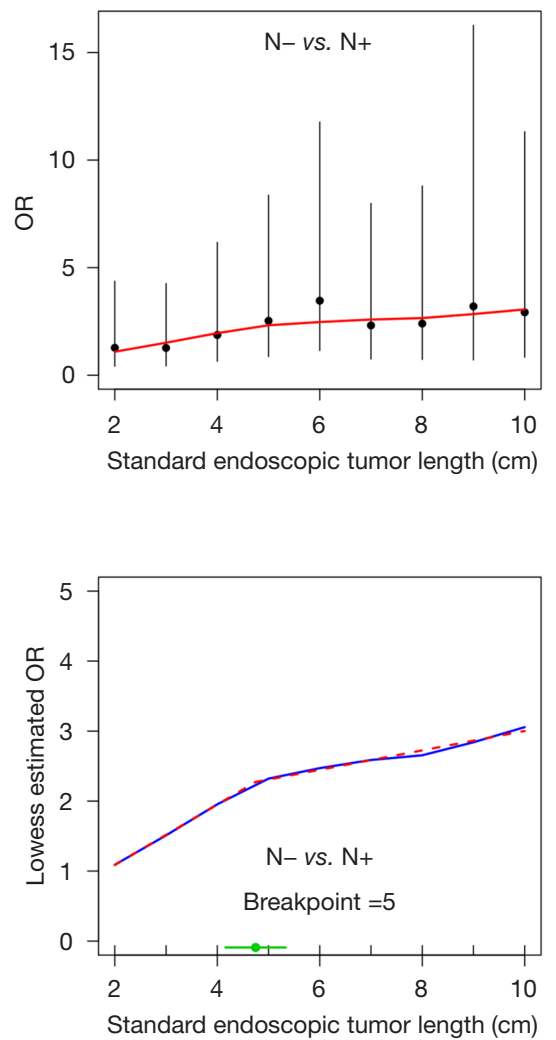

C
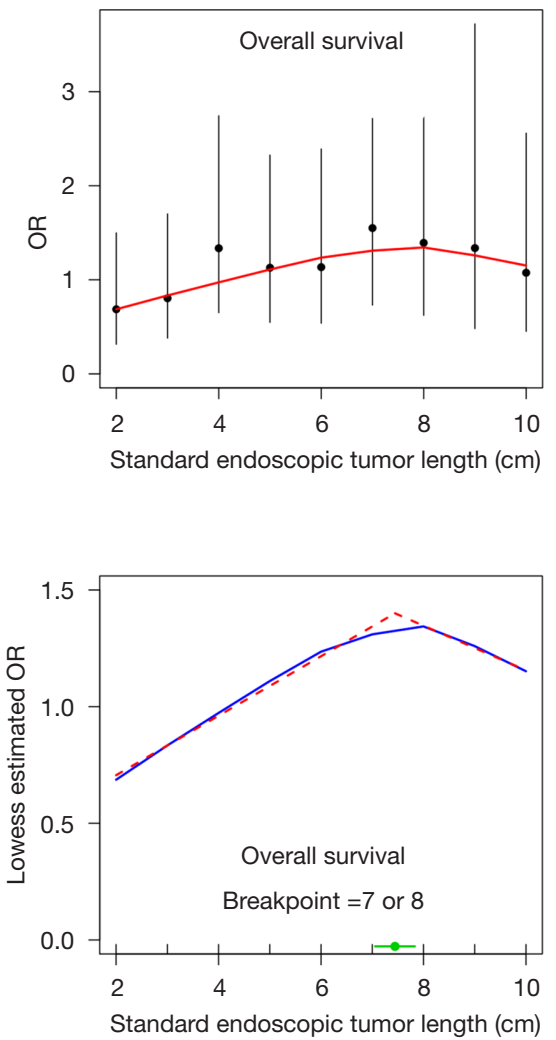

Figure 1 LOWESS fitting curves and determination of structural breakpoints of standard endoscopic tumor length using the Chow test. (A) $\mathrm{pT}$ status, (B) $\mathrm{pN}$ status, and (C) overall survival were evaluated by logistic regression.

The multivariate Cox analysis demonstrated that patients with an ETL $\geq 7.0 \mathrm{~cm}$ had a significantly elevated risk of mortality (adjusted HR, 1.335; 95\% CI, 1.004-1.774) after adjustment for other prognostic factors.

\section{Clinical nomogram for $O S$}

A nomogram incorporating the aforementioned significant prognostic factors including gender, age, tumor location, T stage, $\mathrm{N}$ stage, length group and so on was constructed (Figure 3A). Each subtype within these variables was assigned a score on the point scale (Table S1). By summing the scores of each prognostic factor, we obtained the nomogram score for each patient (median, 191.3; range, 83.4-296.3). The calibration plots showed an excellent agreement between 1-, 3 -, and 5-year OS predicted by the nomogram prediction and that actually observed (Figure 3B). The c-index value was 0.688 .

\section{Discussion}

This study investigated the role of ETL in patients with resected ESCC based on data from a single institution. A longer ETL was significantly associated with advanced pT status and shorter survival, and $7.0 \mathrm{~cm}$ was identified as the optimal breakpoint.

Several previous studies have evaluated the role of tumor length in patients with esophageal cancer. In the majority of these studies, tumor length was considered as a tumor diameter measured from surgical specimens, known as pathological tumor length (PTL). These studies suggested PTL to be a negative prognostic factor for patients with esophageal cancer (11-14,21). The TNM staging system once considered PTL as a staging parameter for esophageal cancer; however, this idea was abandoned in 1987 (22).

So far, the role of ETL has not been well illuminated. To the best of our knowledge, Valmasoni et al. were the first 


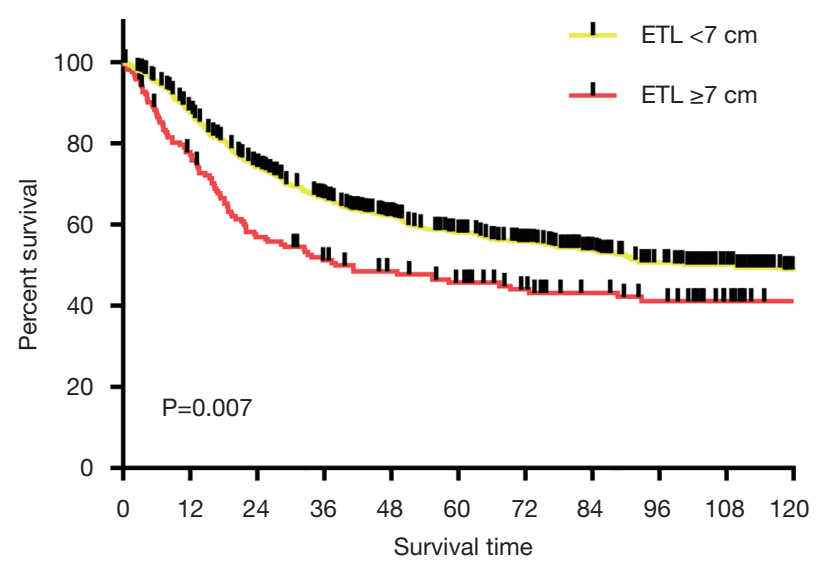

Figure 2 Stratification of overall survival among patients with resected esophageal squamous cell carcinoma at the cut point of the endoscopic tumor length $(7.0 \mathrm{~cm})$.

Table 2 Association between endoscopic tumor length and tumor, node, metastasis staging system

\begin{tabular}{lccc}
\hline \multirow{2}{*}{ Variables } & \multicolumn{2}{c}{ Endoscopic tumor length $(\mathrm{cm})$} & \multirow{2}{*}{ P value $^{\mathrm{a}}$} \\
\cline { 2 - 3 } pT status & $<7$ & $\geq 7$ & 0.098 \\
T1-2 & $201(33.8)$ & $33(26.2)$ & \\
T3-4 & $394(66.2)$ & $93(73.8)$ & \\
pN status & & & 0.030 \\
N0 & $304(51.1)$ & $51(40.5)$ & \\
N1-3 & $291(48.9)$ & $75(59.5)$ & \\
Stage & & & 0.032 \\
I & $75(12.6)$ & $12(9.5)$ & \\
II & $270(45.4)$ & $45(35.7)$ & \\
III & $250(42.0)$ & $69(54.8)$ & \\
\hline
\end{tabular}

${ }^{\mathrm{a}}$, Pearson's $\chi^{2}$ test. pT status, pathological tumor status; $\mathrm{pN}$ status, pathological nodal status.

and only researchers to report longer ETL to be a negative prognostic factor in esophageal cancer (10), and the findings of the present study are consistent with this.

It has been reported that the most common breakpoint for tumor length is $3.0 \mathrm{~cm}$ (12). The cutoff value of $7.0 \mathrm{~cm}$ has not been applied before, which might largely be due to the following reasons. First, as stated above, most studies to date have reported on PTL (11-13). Second, squamous cell carcinoma was intermixed with adenocarcinoma as the
Table 3 Multivariate analysis of overall survival

\begin{tabular}{|c|c|c|c|}
\hline Variable & aHR & $95 \% \mathrm{Cl}$ & $\mathrm{P}$ \\
\hline \multicolumn{4}{|l|}{ Sex } \\
\hline Male & 1 & & \\
\hline Female & 0.765 & $0.603-0.971$ & 0.028 \\
\hline Age & 1.021 & $1.002-1.025$ & 0.019 \\
\hline No. of nodes dissected & 0.984 & $0.977-0.990$ & $<0.001$ \\
\hline \multicolumn{4}{|l|}{ pT status } \\
\hline $\mathrm{T} 1$ & 1 & & \\
\hline T2 & 2.063 & $1.234-3.449$ & 0.006 \\
\hline T3 & 2.692 & $1.664-4.354$ & $<0.001$ \\
\hline $\mathrm{T} 4$ & 5.393 & $2.958-9.834$ & $<0.001$ \\
\hline \multicolumn{4}{|l|}{ pN status } \\
\hline No & 1 & & \\
\hline $\mathrm{N} 1$ & 1.721 & $1.372-2.159$ & $<0.001$ \\
\hline N2 & 3.135 & $2.409-4.080$ & $<0.001$ \\
\hline N3 & 4.166 & $2.810-6.177$ & $<0.001$ \\
\hline \multicolumn{4}{|l|}{ Tumor location } \\
\hline Upper esophagus & 1 & & \\
\hline Middle esophagus & 0.821 & $0.630-1.069$ & 0.143 \\
\hline Lower esophagus & 0.636 & $0.468-0.865$ & 0.004 \\
\hline \multicolumn{4}{|l|}{ Tumor length (cm) } \\
\hline$<7$ & 1 & & \\
\hline$\geq 7$ & 1.335 & $1.004-1.774$ & 0.047 \\
\hline
\end{tabular}

research object in most previous studies (10-14). Third, different research concepts require different statistical methods, which might yield different cutoff points. For instance, in the evaluation of the impact of tumor length on long-term survival, breakpoints have commonly been defined as Cut-off point, such as the median value $(3.0 \mathrm{~cm})$ by Yendamuri et al. (12), the tertiles $(3.0$ and $4.5 \mathrm{~cm}$ ) by Hollis et al. (14), and the 4 milestone points (2.0, 4.0, 6.0, and $8.0 \mathrm{~cm}$ ) by Haisley et al. (11). In another study, the breakpoint was defined as the point with the highest capacity for long-term survival stratification; thus, regression tree survival analysis was conducted (10). Still, in this study, we hypothesized that ETL could potentially be indicative of $\mathrm{pT}$ status, $\mathrm{pN}$ status, and prognosis. Accordingly, the 


\section{A}

Points
Gender
Age
Node.dissection
Tstage
Nstage
Location
Lengthgroup
Total points
1 year survival probability
3 year survival probability
5 year survival probability

B

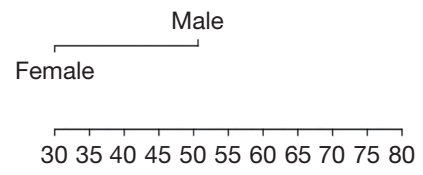

3035404550556065707580
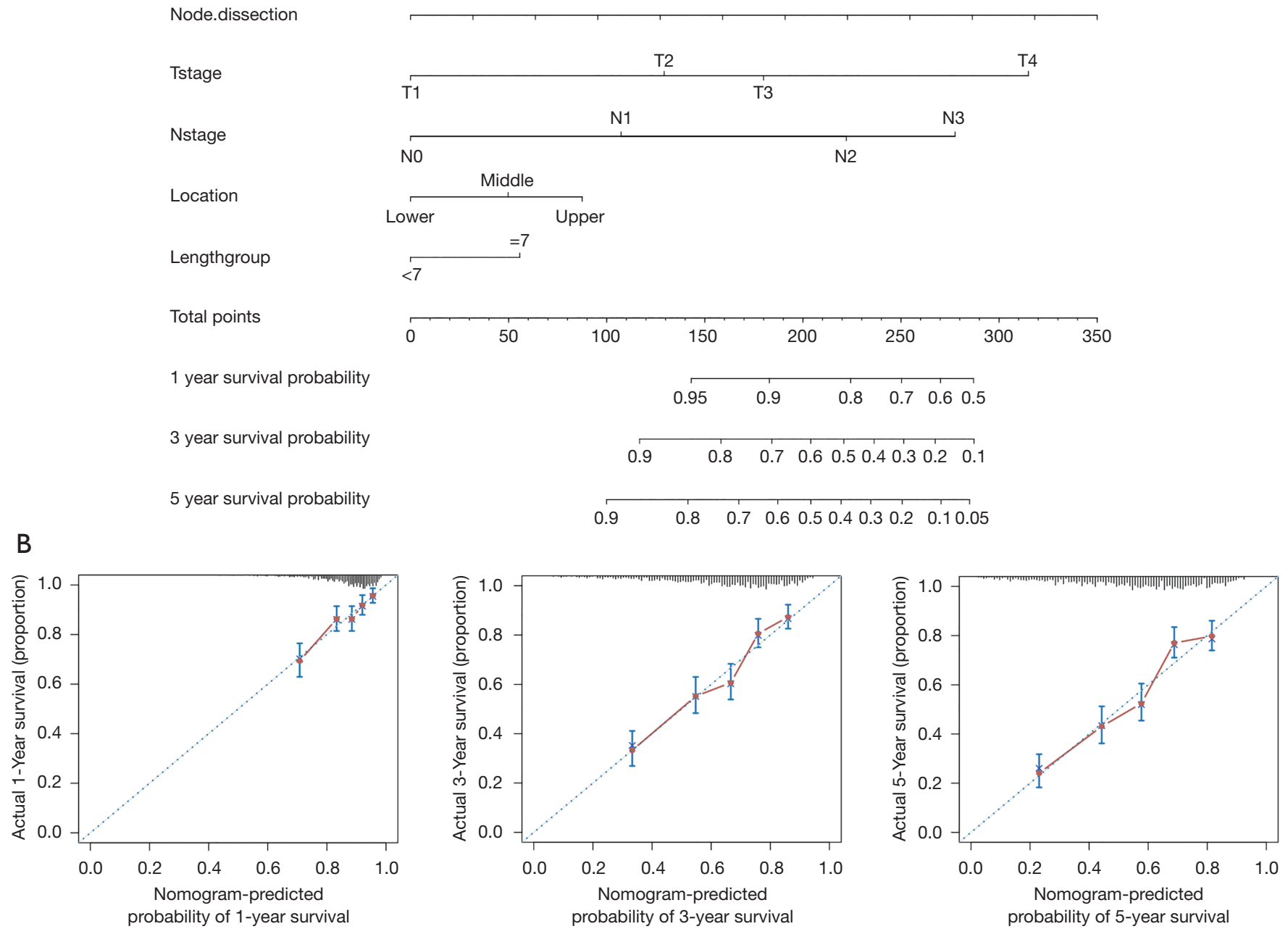

Figure 3 Nomogram incorporating the significant prognostic factors including gender, age, tumor location, $\mathrm{T}$ stage, $\mathrm{N}$ stage, length group and so on was constructed. (A) Nomogram to predict overall survival in patients with resected esophageal squamous cell carcinoma. The calibration curves for predicting patient survival at each time point. (B) Nomogram-predicted overall survival is plotted on the $\mathrm{x}$-axis, and actual overall survival is plotted on the $y$-axis. A plot along the $45^{\circ}$ line would indicate a perfect calibration model in which the predicted probabilities are identical to the actual outcomes.

breakpoint was defined as the inflection point by which to distinguish risk trends for these parameters. For this purpose, we firstly rounded the exact value of the ETL to the nearest whole number, the so-called SETL. The OR of "T3-4/T1-2" and "N+/N-", and the HR of OS were calculated for each SETL. Then, the risk curves of $\mathrm{pT}, \mathrm{pN}$, and OS were plotted with reference to these scatter points, and the breakpoint was defined as the best knee point of each curve using the Chow test. Finally, $7.0 \mathrm{~cm}$ was selected as the cutoff value due to its excellent capacity for stratifying 
most risk curves. We believe that the breakpoint yielded through this method can accurately reflect the authentic associations of ETL with pT status, $\mathrm{pN}$ status, and OS.

Based on our observations, we believe ETL to be an optimal indicator of the longitudinal scope of a primary tumor. The impact of ETL should be further explored in ESCC for the following reasons. First, ETL is significantly associated with pT status. In this study, the frequency of advanced T (T3-T4) status was higher among patients with an ETL $\geq 7.0 \mathrm{~cm}$ than those with an ETL $<7.0 \mathrm{~cm}(73.8 \%$ vs. $66.2 \%, \mathrm{P}=0.098$ ) (Table 2). Therefore, the ETL might assist in the accurate clinical staging of primary tumors. Second, the detrimental prognostic impact of the ETL is independent of $\mathrm{p} T$ status. In this study, after adjustment for confounders, the risk of mortality for patients with an ETL $\geq 7.0 \mathrm{~cm}$ was $30 \%$ higher than that for the ETL $<7.0 \mathrm{~cm}$ group (adjusted HR, 1.335; 95\% CI, 1.004-1.774). Therefore, a longer tumor length $(\mathrm{ETL} \geq 7.0 \mathrm{~cm}$ ) should be considered as an indicator of advanced primary tumor. Nevertheless, in this study, we failed to prove a significant association between ETL and nodal status, although other studies have reported an elevated risk of node metastasis among patients with longer tumors (9).

In this study, a clinical nomogram based on ETL and other proven independent prognostic factors was established. Calibration plots showed good agreement between predicted OS and that actually observed. The c-index of our nomogram was larger than that of the $8^{\text {th }}$ AJCC staging system (0.688 vs. $0.660, \mathrm{P}=0.032)$, revealing more information regarding the patient outcome and more accurately reflecting patient outcome. This finding provided further evidence of the reliability and homogeneity of our results (Table 2).

The main limitation of this study is that it did not include patients with endoscopically impassable tumor stenosis. In fact, when the study was initiated, we proposed using the concept of so-called transluminal tumor length (TTL). For endoscopically passable ESCC, TTL was determined based on endoscopic results. For patients who did not undergo preoperative endoscopic examination or those with endoscopically impassable ESCC, TTL was comprehensively determined on the basis of barium swallow test or computed tomography examination. Finally, 927 patients were recruited, including 34 patients (3.6\%) with impassable tumor stenosis and 172 patients (18.6\%) without endoscopic results. In these excluded $206(34+172)$ patients, we failed to observe any association between transluminal tumor length and $\mathrm{pT}$ status $(\mathrm{P}=0.212), \mathrm{pN}$ status $(\mathrm{P}=0.770)$, or prognosis $(\mathrm{P}=0.114)$. We speculate that these observations might be attributable to 2 reasons. First, the results of barium swallow test or computed tomography examination might introduce bias to the calculation of transluminal tumor length. Second, the impact of transluminal tumor length might be lessened in patients with endoscopically impassable tumor stenosis. Therefore, the findings of our study cannot be directly transferred to patients without preoperative endoscopy examination or those with endoscopically impassable ESCC. In addition, this is a retrospective study based on data from a single institution during a long study period. Although we conducted multivariate analysis to minimize confounders associated with heterogeneity, further validation in a dataset from another institution are needed to further verify our results.

In conclusion, longer tumor length, with a breakpoint of ETL $\geq 7.0 \mathrm{~cm}$, representing the extended longitudinal scope of the primary tumor, is a negative prognostic factor for patients with ESCC. Validation of our findings in a multicenter prospective study is still needed.

\section{Acknowledgments}

Funding: None.

\section{Footnote}

Reporting Checklist: The authors have completed the STROBE reporting checklist. Available at http://dx.doi. org/10.21037/jtd-21-108

Data Sharing Statement: Available at http://dx.doi. org/10.21037/jtd-21-108

Conflicts of Interest: All authors have completed the ICMJE uniform disclosure form (available at http://dx.doi. org/10.21037/jtd-21-108). The authors have no conflicts of interest to declare.

Ethical Statement: The authors are accountable for all aspects of the work in ensuring that questions related to the accuracy or integrity of any part of the work are appropriately investigated and resolved. Ethical approval was obtained from the review board committee of Fujian Cancer Hospital. The study was conducted in accordance 
with the Declaration of Helsinki (as revised in 2013). Because of the retrospective nature of the research, the requirement for informed consent was waived.

Open Access Statement: This is an Open Access article distributed in accordance with the Creative Commons Attribution-NonCommercial-NoDerivs 4.0 International License (CC BY-NC-ND 4.0), which permits the noncommercial replication and distribution of the article with the strict proviso that no changes or edits are made and the original work is properly cited (including links to both the formal publication through the relevant DOI and the license). See: https://creativecommons.org/licenses/by-nc-nd/4.0/.

\section{References}

1. Bray F, Ferlay J, Soerjomataram I, et al. Global cancer statistics 2018: GLOBOCAN estimates of incidence and mortality worldwide for 36 cancers in 185 countries. CA Cancer J Clin 2018;68:394-424.

2. Mok TS, Wu YL, Thongprasert S, et al. Gefitinib or carboplatin-paclitaxel in pulmonary adenocarcinoma. $\mathrm{N}$ Engl J Med 2009;361:947-57.

3. Matsubara T, Takamori S, Haratake N, et al. The impact of immune-inflammation-nutritional parameters on the prognosis of non-small cell lung cancer patients treated with atezolizumab. J Thorac Dis 2020;12:1520-8.

4. Weber JS, D'Angelo SP, Minor D, et al. Nivolumab versus chemotherapy in patients with advanced melanoma who progressed after anti-CTLA-4 treatment (CheckMate 037): a randomised, controlled, open-label, phase 3 trial. Lancet Oncol 2015;16:375-84.

5. Ando N, Iizuka T, Ide H, et al. Surgery plus chemotherapy compared with surgery alone for localized squamous cell carcinoma of the thoracic esophagus: a Japan Clinical Oncology Group Study--JCOG9204. J Clin Oncol 2003;21:4592-6.

6. Yang H, Liu H, Chen Y, et al. Neoadjuvant Chemoradiotherapy Followed by Surgery Versus Surgery Alone for Locally Advanced Squamous Cell Carcinoma of the Esophagus (NEOCRTEC5010): A Phase III Multicenter, Randomized, Open-Label Clinical Trial. J Clin Oncol 2018;36:2796-803.

7. Zheng Y, Fu S, He T, et al. Predicting prognosis in resected esophageal squamous cell carcinoma using a clinical nomogram and recursive partitioning analysis. Eur J Surg Oncol 2018;44:1199-204.
8. Zhang D, Zheng Y, Wang Z, et al. Comparison of the 7th and proposed 8th editions of the AJCC/UICC TNM staging system for esophageal squamous cell carcinoma underwent radical surgery. Eur J Surg Oncol 2017;43:1949-55.

9. Rice TW, Zuccaro G Jr, Adelstein DJ, et al. Esophageal carcinoma: depth of tumor invasion is predictive of regional lymph node status. Ann Thorac Surg 1998;65:787-92.

10. Valmasoni M, Pierobon ES, Ruol A, et al. Endoscopic Tumor Length Should Be Reincluded in the Esophageal Cancer Staging System: Analyses of 662 Consecutive Patients. PLoS One 2016;11:e0153068.

11. Haisley KR, Hart KD, Fischer LE, et al. Increasing tumor length is associated with regional lymph node metastases and decreased survival in esophageal cancer. Am J Surg 2016;211:860-6.

12. Yendamuri S, Swisher SG, Correa AM, et al. Esophageal tumor length is independently associated with long-term survival. Cancer 2009;115:508-16.

13. Zhang X, Wang Y, Qu P, et al. Prognostic Value of Tumor Length for Cause-Specific Death in Resectable Esophageal Cancer. Ann Thorac Surg 2018;106:1038-46.

14. Hollis AC, Quinn LM, Hodson J, et al. Prognostic significance of tumor length in patients receiving esophagectomy for esophageal cancer. J Surg Oncol 2017;116:1114-22.

15. Rice TW, Kelsen D, Blackstone EH, et al. AJCC Cancer Staging Manual. 8th ed. New York, NY: Springer, 2017:185-202.

16. Liang W, He J, Shen Y, et al. Impact of Examined Lymph Node Count on Precise Staging and Long-Term Survival of Resected Non-Small-Cell Lung Cancer: A Population Study of the US SEER Database and a Chinese MultiInstitutional Registry. J Clin Oncol 2017;35:1162-70.

17. Chow GC. Tests of equality between sets of coefficients in two linear regressions. Econometrica 1960;28:591-605.

18. Zheng $\mathrm{W}$, Huang $\mathrm{Y}$, Chen $\mathrm{H}$, et al. Nomogram application to predict overall and cancer-specific survival in osteosarcoma. Cancer Manag Res 2018;10:5439-50.

19. Liang W, Zhang L, Jiang G, et al. Development and validation of a nomogram for predicting survival in patients with resected non-small-cell lung cancer. J Clin Oncol 2015;33:861-9.

20. Cen D, Xu L, Li N, et al. BI-RADS 3-5 microcalcifications can preoperatively predict breast cancer HER2 and Luminal a molecular subtype. 
Oncotarget 2017;8:13855-62.

21. Arigami T, Uchikado Y, Omoto I, et al. Primary Tumor Score Based on Tumor Depth and Length Predicts Prognosis in Esophageal Squamous Cell Carcinoma. Anticancer Res 2018;38:5447-52.

Cite this article as: Chen P, Zheng Y, He H, Wang PY, Wang F, Liu SY. The role of endoscopic tumor length in resected esophageal squamous cell carcinoma: a retrospective study. J Thorac Dis 2021;13(1):353-361. doi: 10.21037/jtd-21-108
22. Hutter RV. At last--worldwide agreement on the staging of cancer. Arch Surg 1987;122:1235-9.

(English Language Editor: J. Reynolds) 
Supplementary

Table S1 The computational formula of the nomogram score for each prognostic factor

\begin{tabular}{ll}
\hline Variables & The computational formula of nomogram score \\
\hline Sex & Male $=15.1 ;$ female $=0$ \\
Age & $0.732766261 \times$ age -21.982987824 \\
Node dissection & $100-0.909090909 \times$ node dissection \\
PT status & $\mathrm{T} 1=0 ; \mathrm{T} 2=36.9 ; \mathrm{T} 3=51.4 ; \mathrm{T} 4=90.0$ \\
pN status & $\mathrm{N} 0=0 ; \mathrm{N} 1=30.7 ; \mathrm{N} 2=63.5 ; \mathrm{N} 3=79.3$ \\
Tumor location & Upper $=25.0 ;$ middle $=14.2 ;$ lower $=0$ \\
Endoscopic tumor length & $\leq 7 \mathrm{~cm}=0 ;>7 \mathrm{~cm}=15.9$ \\
\hline
\end{tabular}

pT status, pathological tumor status; pN status, pathological nodal status. 